shown that a limited number of infective bites makes individuals in malaria-endemic regions more susceptible to subsequent malaria infection as they grow older due to waning or lost immunity. This study investigated the evolution of malaria morbidity for 14 years in Korogwe, Tanzania since 2003.

Methods A longitudinal study was carried out in Korogwe over 14 years, from January 2003 to December 2017 whereby community health workers (CHWs) passively monitored malaria episodes at a village health post. They evaluated febrile episodes and collected blood smears from all residents of the community who presented with fever. The blood smears were processed and read at the Korogwe field station by two independent microscopists. Artemisinin combination therapy (ACT) and malaria rapid diagnostic tests were introduced in the community in 2007. Uncomplicated malaria cases were treated with sulphadoxine-pyrimethamine (SP) from 2003 to 2006, then with artemether-lumefantrine (ALu) from 2007. Results A total of 20,841 attendances were documented by CHWs between 2003 and 2017. Malaria parasitaemia was documented in 5043 consultations [24.1\% (95\% confidence interval (CI): $23.6 \%$ to $24.8 \%$ )]. Interestingly, malaria episodes declined markedly from $38.12 \%$ to $10.42 \%$ between 2003 and 2017. The highest reduction was documented in 2010 (at $3.1 \%$ ) but thereafter, there was an increase in malaria in 2015 to $32.2 \%$ which decreased to $10.42 \%$ in 2017 . Use of longlasting insecticide-treated nets (LLINs) was associated with reduction of malaria episodes by $34 \% \quad(95 \%$ CI: $26 \%$ to $42 \%)$.

Conclusion Prompt diagnosis at village level, use of ACT and LLINs has contributed to the reduced number of malaria episodes in Korogwe. However, the malaria resurgences raised concerns about malaria elimination in these communities.

\section{PO 8576 EFFECT OF IVERMECTIN TREATMENT ON THE FREQUENCY OF SEIZURES IN PERSONS WITH ONCHOCERCIASIS-ASSOCIATED EPILEPSY: PRELIMINARY RESULTS OF A RANDOMISED CLINICAL TRIAL}

\begin{abstract}
${ }^{1}$ Mandro Ndahura*, ${ }^{2}$ Deby Mukendi, ${ }^{3}$ Françoise Nyisi, ${ }^{4}$ Deogracias Rossy, ${ }^{5}$ Joseph Siewe, ${ }^{3}$ Germain Abhafule, ${ }^{5}$ Swabra Nakato, ${ }^{6}$ Patrick Suykerbuyk, ${ }^{7}$ Joseph Siewe, ${ }^{7}$ An Hotterbeekx, ${ }^{7}$ Robert Colebunders, ${ }^{7}$ Sonia Menon. ${ }^{1}$ Ministry of Health of Ituri Province, Democratic Republic of the Congo; ${ }^{2}$ University of Kinshasa, Democratic Republic of the Congo; ${ }^{3}$ Centre de Recherche en Maladies Tropiclales de I'Ituri/Hopital de Rethy, Democratic Republic of the Congo; ${ }^{4}$ Programme National de Maladies Tropicales Négligées/Ituri Sud, Democratic Republic of the Congo; ${ }^{5}$ Global Health Institute, University of Antwerp, Belgium; ${ }^{6}$ Fondation Damien, Brussels, Belgium; ${ }^{7}$ Global Health Institute, University of Antwerp, Belgium
\end{abstract}

\subsection{6/bmjgh-2019-EDC.150}

Background Many studies reported an association between epilepsy and onchocerciasis. Moreover, anecdotal evidence suggests that ivermectin may reduce seizure frequency in persons with onchocerciasis-associated epilepsy (PWOAE). Therefore, we performed a randomised clinical trial among ivermectinnaïve persons with epilepsy in onchocerciasis-endemic villages in the Ituri province, Democratic Republic of the Congo.

Methods PWOAE were randomised in an arm receiving immediate ( $\operatorname{arm~A)}$ or delayed (4 months later) ivermectin treatment (arm B). All participants were receiving anti-epileptic drugs. Inclusion criteria were: age $>5$ years, signed informed consent, normal neurological development until onset of epilepsy between 5-18 years of age, seizure frequency of $\geq 2$ seizures/month, presence of microfilaria in skin snip and/or antibodies against the Ov16 antigen. Primary study outcome: seizure freedom at month 4; secondary outcome: $>50 \%$ reduction in seizure frequency at month 4 compared to reported seizure frequency at randomisation. The proposed sample size was 110 PWOAE.

Results 93 PWOAE, 57 males and 36 females, (mean age 22), were enrolled between October and November 2017. On March 2018, 90 (97\%) participant completed their $4^{\text {th }}$-month evaluation. One serious adverse event was observed during the trial (Steven Johnson reaction caused by phenobarbital). Considering all participants there was no significant difference in outcome between the 2 arms. However, considering participants with presence of microfilariae at enrollment, at month 4, 26/39 (66.6\%) in arm A and 20/44 (45.5\%) in arm B were seizure free $(p=0.05)$ and a $50 \%$ reduction of the number of seizures was observed in $9 / 39(23.1 \%)$ in arm $\mathrm{A}$ and $7 / 43$ $(16.3 \%)$ in arm B. $(\mathrm{p}=0.4)$.

Conclusion Ivermectin may have an added value in reducing the frequency of seizures in PWOAE treated with anti-epileptic drugs. However, a larger study is needed to confirm this.

\section{PO 8578 THE ROLE OF CLINICAL RESEARCH IN EDUCATING THE HEALTH WORKFORCE IN GUINEA-BISSAU}

Isaquel B Silva. Bandim Health Project, Bissau, Guinea-Bissau

\subsection{6/bmjgh-2019-EDC.151}

Background The World Health Organisation estimates a global deficit of approximately 4.3 million health workers, particularly doctors, nurses and midwives. Guinea-Bissau is among the 36 countries that suffer from a critical need for human resources (less than 23 health professionals per 10.000 inhabitants). The present research investigates the impact of clinical trials on health worker's capacity and training.

Methods This is a qualitative study. I interviewed health professionals who assisted in clinical trials during the past five years and obtained assistance to attend health-related courses. The interviews collected data about the professional's technical capacity, clinical practices, work conditions, their qualification for their work and socio-economic variables. Additionally, documents about clinical trials conducted in Guinea-Bissau through North-South cooperation, were analysed.

Results Interviews were held with 35 health professionals (21 female, 14 male) who participated in clinical trials with the Bandim Health Project (Guinea-Bissau) as research assistants and who received subsidies to realise undergraduate or graduate degrees. Among those interviewed, 28 (80\%) received support to realise an undergraduate degree in nursing, 4 (11\%) a laboratory course, and $3(9 \%)$ a postgraduate course. Twentyfour $(24 ; 69 \%)$ stated that they experienced significant improvements in their working conditions within the institutions where they worked after their clinical trials; 7 (20\%) declared that they have progressed in their career after being placed in a health center or a hospital; 29 (83\%) stated that the participation in clinical trials significantly strengthened their technical capacity and had a positive impact on their careers.

Conclusion I observed a positive impact of the involvement in clinical research on the development of health professionals' capacity. However, the quantity of clinical research in GuineaBissau is insufficient to train all health practitioners in this area. 